\title{
Procurement Auctions Using Actor-Critic Type Learning Algorithm
}

\author{
C. V. L. Raju \\ Department of CSA \\ Indian Institute of Science \\ Bangalore, India \\ raju@csa.iisc.ernet.in
}

\author{
Y. Narahari \\ Department of CSA \\ Indian Institute of Science \\ Bangalore, India \\ hari@csa.iisc.ernet.in
}

\author{
Saurabh Shah \\ Department of CSIS \\ BITS, Pilani, India \\ sssaurabh2k@yahoo.co.in
}

\begin{abstract}
Procurement, the process of obtaining materials or services, is a critical process for any organization. While procuring a set of items from different suppliers who may sell only a subset (bundle) of a desired set of items, it will be required to select an optimal set of suppliers who can supply the desired set of items. This is the optimal vendor selection problem. Bundling in procurement has benefits such as demand aggregation, supplier aggregation, and lead time reduction. The NP. hardness of the vendor selection problem motivates us to formulate a compatible linear programming problem by relaxing the integer constraints and imposing additional constraints. The newly formulated problem can be solved by a novel iterative algorithm proposed recently in the literature. In this paper, we show that the application of this iterative algorithm will lead to an iterative procurement auction that improves the efficiency of the procurement process. By using reinforcement learning to orchestrate the iterations of the algorithm, we show impressive gains in computational efficiency of the algorithm.
\end{abstract}

Keywords: eProcurement, iBundle, Primal=Dual algorithm, Stochastic Dynamic Programming, Q-learning, Actor-Critic algorithm.

\section{Introduction}

Many companies world-wide are continuously looking to improve supply chain efficiencies. A major way of obtaining these efficiencies is through the use of e-marketplaces. Despite slower economic growth in the first two quarters of 2001 , it is expected that emarketplaces will provide tremendous value and efficiency to supply chain operations. An e-marketplace is a many-to-many market mechanism which allows an enterprise to be connected to its own group of business partners using its application and network infrastructure to optimize various business processes. Marketplaces provide high performance and tight integration with business partners. Procurement (direct and indirect) is an extremely important business process that can benefit from e-marketplaces. Private Marketplaces are now emerging as the preferred platforms for e-procurement. In this case, the buyers are all from the same company which owns the private marketplace. Individual buyers are possibly from different divisions of the same company. The sellers are the preferred suppliers for this company.

The concept of bundling has the potential of improving the efficiency of the procurement process. Here, related items are bundled into a set of items and the vendors are allowed to bid for the entire set or a subset of the items. This can result in exploitation of complementarity of products and in achieving logistics and lead time efficiencies. The allocation problem (the problem of selecting which vendors will supply which bundles), however, becomes hard, in fact NP-hard.

In this paper, we look into the following procurement problem. There is a buyer who needs to procure a bundle of items, say $\left\{A_{1}, \ldots, A_{n}\right\}$. There is a set of sellers who can supply subsets of this bundle. The buyer engages the sellers in an iterative bundle auction with the objective of minimizing his cost of procuring the bundle of items.

We solve this problem by first formulating the problem as a linear integer programming problem that is NP-hard. We then formulate a relaxed version of this problem and show its connection to the iBundle algorithm of David Parkes [1]. This leads to what we call as the iterative bundle procurement auction (IBPA). We observe that the use of Parkes' algorithm has inherent computational complexities in solving IBPA. We then propose the use of reinforcement learning techniques in reducing the computational complexity of solving IBPA.

\subsection{Review}

Auctions allow selling goods and apply in situations where a more conventional market in which buyers consider the price as given, does not exist. When an Auction of multiple items is performed, it is often 
desirable to allow bids on combination of items, as opposed to only on single items. Such an auction is called Combinatorial, and the exponential number of possible combinations results in computational intractability of many aspects regarding such an auction. In particular, the auctioneer's winner-determination (WD) problem, that is choosing bids to optimize the cost or revenue is $N P$-hard. In iBundle [1] the auctioneer solves a sequence of WD problems to maintain a provisional allocation, which are small in size. At the end of the auction the provisional allocation will be the permanent allocation. iBundle algorithm is a variant of traditional primal-dual algorithm to solve the linear programme formulation of Bickchandani and Ostroy's package assignment model [2].

More recently, electronic commerce has generated new interest in investigating how adoptive software agents may utilize reinforcement learning algorithms such as Q-learning to make economic decisions such as setting prices in a competitive market place. Price control in a Dutch auction [6], pricebots and shopbots [5] are some examples. In this paper we use reinforcement learning technique to iBundle algorithm for choosing minimum bid increment to reduce the number of auction rounds to be conducted and economy loss.

\section{An Overview of Reinforcement Learning}

\subsection{Reinforcement Learning}

The term reinforcement learning (RL) originates in studies of learning behavior of animals. Mathematically, it falls somewhere between the supervised and unsupervised learning paradigms of pattern recognition. $\mathrm{RL}$ neither calls for exact information about error from the environment, nor works with no information from the environment. RL expects a "reinforcement signal" from the environment indicating whether or not the latest move is in the right direction. RL procedures have been established as powerful and practical methods for solving Markovian Decision Problems (MDP).

\subsubsection{MDP}

Consider a process, observed at time epochs $t=0,1$, $\ldots$, to be in one of the states $i \in \mathrm{S}$. After observing the state of the process an action $a \in A=\left\{a^{1}, a^{2}, \ldots, a^{m}\right\}$ is taken, where $A$ is the set of all possible actions. If the process is in state $i$ at time $n$ and action $a$ is chosen, then two things occur, (1) we incur a cost/reward $\mathrm{R}(i, a)$ (2) the next state of the system is chosen according to the transition probabilities $P_{i j}(a)$. If we let $X_{n}$ denote the process at time $n$ and $a_{n}$ the action chosen at that time, then assumption (2) can be stated as:

$$
\begin{aligned}
P_{i j}(a)= & P\left(X_{n+1}=j \mid X_{0},\right. \\
& \left.a_{0}, X_{1}, a_{1}, \ldots, X_{n}=i, a_{n}=a\right)
\end{aligned}
$$

We suppose that we always work with the case where $|R(i, a)|<M \forall i$,a. A policy is any rule for choosing actions. An important subclass of policies is the class of stationary policies. A policy is said to be stationary if the action it chooses at time $n$ only depends on the state of the process at time $n$, so stationary policy is a function $\pi: S \rightarrow A$. If policy $\pi$ is employed, then $\left\{X_{n}, n=0,1, \ldots\right\}$ is a Markov chain with transition probabilities $P_{i j}(\pi(i))$, it is for this reason the process is called a Markov decision process. A stationary randomized policy can be considered as a map $\varphi: S \rightarrow \mathcal{P}(A)$ $(\mathcal{P}(\ldots)=$ the space of probability vectors on "..."), which gives the conditional probabilities of $a^{j}$ given $X_{n}$ for all $1 \leq j \leq m$.

To determine policies that are in some sense optimal, we consider the infinite horizon discounted return as our optimal criterion. This criterion assumes a discount factor $\alpha, 0<\alpha<1$, and among all policies $\pi$, attempts to maximize $V^{\pi}$ where

$$
V^{\pi}(i)=E^{\pi}\left[\sum_{n=0}^{\infty} R\left(X_{n}, a_{n}\right) \alpha^{n} \mid X_{0}=i\right], \quad i \in S
$$

The function $V^{\pi}: X \rightarrow \mathcal{R}$ is called the value function for policy $\pi$. The use of a discount factor is economically motivated by the fact that the value of money earned tomorrow is worth discounted amount today. The optimal value of the value function, is:

$$
V^{*}(i)=\max _{\pi} V^{\pi}(i), \quad i \in S
$$

An important equation that $V^{*}$ satisfies is Bellman's optimality equation[?]:

$$
V(i)=\max _{a}\left[R(i, a)+\alpha \sum_{j} P_{i j}(a) V(j)\right], \quad i \in S
$$

The fact that $V^{*}$ satisfies Bellman's equation can be explained as follows. In the above equation the term in square brackets on the right hand side is the total reward that one would get if action $a$ is chosen at the first time step and then the system performs optimally in all future time steps. Clearly, this term cannot exceed $V^{*}(i)$ since that would violate the definition of $V^{*}$, thus $V^{*}$ satisfies the Bellman's equation. it is also known that Bellman's equation has a unique solution [?]. Now the optimal decision problem turns out to be finding $V^{*}$.

\subsubsection{DP Techniques}

Two standard approaches to compute $V^{*}$ are value iteration and policy iteration.

Value iteration: This starts with an initial guess $V_{0}$ for the optimal $V^{*}$ and recursively iterates as per

$$
V_{n+1}(i)=\max _{a}\left[R(i, a)+\alpha \sum_{j} P_{i j}(a) V_{n}(j)\right], i \in S
$$


for $n \geq 0$. Using Banach contraction mapping theorem, it is easy to show that $V_{n} \rightarrow V$ at an exponential rate. Policy iteration: This starts with initial stationary (randomized) policy $\pi_{0}: S \rightarrow A$, for an optimal policy, it does iteratively for $n \geq 0$ as follows:

Step 1: Given $\pi_{n}($.$) , find V_{n}: S \rightarrow \mathcal{R}$, satisfying

$$
V_{n}(i)=R\left(i, \pi_{n}(i)\right)+\alpha \sum_{j} P_{i j}\left(\pi_{n}(i)\right) V_{n}(j), i \in S
$$

Step 2 : Find

$$
\pi_{n+1}(i) \in \operatorname{argmax}\left(R(i, .)+\alpha \sum_{j} P_{i j}(.) V_{n}(j)\right), i \in S
$$

then $V_{n} \rightarrow V$ in finitely many steps.

Convergence issues: In value iteration, we solve the nonlinear system of equations (5). Define the nonlinear value iteration operator, $B$, in vector-form as

$$
B(V)=\max _{\pi}(R(\pi)+\alpha P(\pi) V), V \in \mathcal{R}^{|S|}
$$

Component wise, $B$ can be written as follows:

$$
B_{i}(V)=\max _{a}\left[R(i, a)+\alpha \sum_{j} P_{i j}(a) V(j)\right], i \in S .
$$

For $\alpha<1, B$ is a contraction operator because $\forall V \in$ $\mathcal{R}^{|S|},\left\|B(V)-V^{*}\right\| \leq \alpha\left\|V-V^{*}\right\|$. Therefore, the value iteration algorithm can be proven to converge to $V^{*}$ by using Banach contraction mapping theorem, and $V_{n} \rightarrow$ $V$ at an exponential rate.

In policy iteration, we evaluate a fixed stationary policy $\pi$, which requires solving a linear system of equations (6). Here also, we can define an operator $B^{\pi}$ as

$$
B^{\pi}(V)=R(\pi)+\dot{\alpha} P(\pi) V
$$

For $\alpha<1$, the operator $B^{\pi}$ is a contraction operator because $\forall V \in \mathcal{R}^{|S|},\left\|B^{\pi}(V)-V^{\pi}\right\| \leq \alpha\left\|V-V^{\pi}\right\|$. So $V_{\pi}$ is the unique solution to $V=B_{\pi}(V)$. Of course, the operators $B$ and $B^{\pi}$ require knowledge of the state transition probabilities. For getting more insight on the above issues, refer [?], [?]. One has to have complete knowledge of transition probabilities $P_{i j}(a)$ in order to solve the MDP problem by applying the above techniques. When we do not know the environment dynamics (i.e. transition probabilities) we can use reinforcement learning algorithms like Q-learning [?], actor-critic algorithm [?], which are stochastic approximation counterparts of value iteration and policy iteration, respectively. These algorithms are used when the transition probabilities $P_{i j}(a)$ are not explicitly available but a transition with a prescribed probability can be simulated. We now discuss the intuition behind appropriate simulation-based versions of the above two algorithms, for more insights one can refer [?]. The first, of course, is that $P_{i j}(a)$ is replaced by a simulated transition as per the prescribed probabilities $P_{i j}(a)$. In order for this to work, the algorithms should do some averaging. This is ensured by using an incremental version which makes only a small change in current iterates at each step, weighted by a stochastic approximation-like decreasing step size. Qlearning is a simulation-based version of the value iteration, where one works not with value function $V$, but the $\mathrm{Q}$-value defined by

$$
Q(i, a)=R(i, a)+\alpha \sum_{j} P_{i j}(a) V(j), i \in S, a \in A .
$$

Thus $Q$ satisfies

$$
Q(i, a)=R(i, a)+\alpha \sum_{j} P_{i j}(a) \max _{b} Q(j, b)
$$

The iterative scheme is

$$
Q_{n+1}(i, a)=R(i, a)+\alpha \sum_{j} P_{i j}(a) \max _{b} Q_{n}(j, b)
$$

If $P_{i j}(a)$ are not available, in Q-learning one replaces the above equation by

$$
\begin{aligned}
Q_{n+1}(i, a)= & \left(1-\gamma_{n}\right) Q_{n}(i, a)+\gamma_{n}\left(R\left(i, a, \xi_{n}(i, a)\right)\right. \\
& +\alpha \max _{b} Q_{n}\left(\xi_{n}(i, a), b\right)
\end{aligned}
$$

we choose a sequence of step sizes $\gamma_{n}$ such that $\sum \gamma_{n}=$ $\infty$ and $\sum \gamma_{n}^{2}<\infty$ and $\xi_{n}(i, a)$ is a random variable with law $P_{i .}(a), \gamma_{n}$ is the step size at the $n^{\text {th }}$ iteration. We start the algorithm by initializing the $\mathrm{Q}$ function and by simulation we get $\xi_{n}(i, a)$ and we calculate $R\left(i, a, \xi_{n}(i, a)\right)$, iteratively we update the $Q$ function. Observe that the Q-learning algorithm demands a reinforcement signal, that is $R\left(i, a, \xi_{n}(i, a)\right)$ for learning the $Q$ function, refer [?] for more details of convergence issues of the Q-learning algorithm. Actor-critic type algorithms are simulation-based version of the policy iteration. The linear system of equations in step 1 is replaced by an iterative scheme for its solution

$$
\begin{aligned}
V_{n}^{m+1}= & R\left(i, \pi_{n}(i)\right)+ \\
& \alpha \sum_{j} P_{i j}\left(\pi_{n}^{m}(i)\right) V_{n}^{m}(i), \underline{i} \in S, m \geq \dot{\alpha}(15)
\end{aligned}
$$

where $m$ is being updated for each fixed $n$, the value $V_{n}$ (which is $V_{n}^{m} \rightarrow V_{n}$ ) is then passed to step2 for updating the policy. The crux of the algorithm proposed in [?] is to achieve this two-tier structure by using two different time scales is as follows. We operate with stationary randomized policies rather than stationary policies. Let $\{a(n)\},\{b(n)\}$ be decreasing sequences in $(0,1)$ satisfying $\sum_{n} a(n)=\sum_{n} b(n)=\infty, \sum_{n} a(n)^{2}, \sum_{n} b(n)^{2}<$ $\infty, a(n)=o(b(n))$. Fix $a_{0} \in A$ and let $\Gamma$ denote the projection of $\bar{a}_{n},(r-1)$-vector $\left(\left[\pi\left(i, a^{1}\right), \ldots, \pi\left(i, a^{r}\right)\right]\right)$ onto the simplex $D=\left\{\left[x_{1}, \ldots, x_{r-1}\right] \mid x_{i} \geq 0, \forall i, \sum_{i} x_{i} \leq 1\right\}$. 
The algorithm starts with an initial pair $V_{0}($.$) and$ $\pi_{0}(i, a), i \in S, a \in A \backslash\left\{a_{0}\right\}$, and iterates according to

$$
\begin{aligned}
V_{n+1}(i)= & (1-b(n)) V_{n}(i)+b(n) \sum_{a}\left[R\left(i, a, \xi_{n}(i, a)\right)\right. \\
+ & \left.\alpha V_{n}\left(\xi_{n}(i, a)\right)\right] \pi_{n}(i, a) \\
\pi_{n+1}(i, a)= & \pi_{n}(i, a)+a(n) \sum_{a}\left[R\left(i, a, \xi_{n}(i, a)\right)\right. \\
& +\alpha V_{n}\left(\xi_{n}(i, a)\right) \pi_{n}(i, a)- \\
& \left.V_{n}(i)\right] \pi_{n}(i, a)
\end{aligned}
$$

Evaluating the value function for a particular policy is considered as criticism and updating the present policy is considered as action, that is why it is called as actorcritic algorithm, refer [?] for convergence issues of the actor-critic algorithm.

\section{Procuring items by conducting reverse auction}

Auctions are popular, distributed and autonomy preserving ways of allocating items among multiple bidders (sellers). Auctioneer (buyer) who wants to procure a lot of items conducts the auction, bidders can bid on combinations of items in the lot to be procured by the auctioneer. Determining the set of winning bids to minimize the total procurement cost is a combinatorial optimization problem, which is NP-hard.

iBundle, is an iterative combinatorial auction algorithm proposed by Parkes[1]. In this paper, we design an iBundle-type of auction for procurement (which we call IBPA) and show how using reinforcement learning, one can solve IBPA efficiently.

\subsection{Modeling the procurement (reverse) auction}

Let $G$ denote the set of items to be procured by conducting auction, and $I$ denote the set of bidders, and $S$ $\subseteq G$ denote the bundle of items. The auction proceeds in rounds, indexed $t \geq 1$. The bidder can place XOR bids.

Each agent $i \in I$ has private value $V_{i S}$ for a bundle $S$, where $S \subseteq G$. The objective of the auctioneer ( buyer ) is allocate the items in a way that he can procure set of items

$G$ at minimum possible cost.

The Integer Programming formulation given bellow shows a standard combinatorial resource allocation problem.

$$
{ }_{x_{i S}}^{\max } \sum_{S \subseteq G} \sum_{i \in I} x_{i S}\left(M-V_{i S}\right)
$$

sub. to

$$
\sum_{S \subseteq G} x_{i S} \leq 1, \quad \forall i
$$

$$
\begin{gathered}
\sum_{S \subseteq G, j \in S} x_{i S} \leq 1, \quad \forall j \\
x_{i S} \in 0,1, \quad \forall i, S
\end{gathered}
$$

Where variable $x_{i S}=1$ if bidder $i$ is allocated bundle $S$, and $x_{i S}=0$ otherwise

Constraints 1 meant for each bidder receives at most one bundle, constraints 2 meant for each item will be procured from at most one bidder. Objective function ensures we get every bundle from the bidder who has low private valuation than the other bidders.

Bikchandani and Ostroy[2] formulate the combinatorial resource allocation problem as a linear program.

Auctioneer (buyer) starts the auction for a lot (set $G$ ) of items by giving high value $M$ for all possible bundles from the lot and decrease while auction is progressing.

Primal

$$
\max _{i S, y_{k}} \sum_{S \subseteq G} \sum_{i \in I} x_{i S}\left(M-V_{i S}\right)
$$

sub. to

$$
\begin{gathered}
\sum_{S \subseteq G} x_{i S} \leq 1, \quad \forall i \\
x_{i S} \leq \sum_{k \ni[i, S]} y_{k}, \quad \forall i, S \\
\sum_{k} y_{k} \leq 1 \\
x_{i S}, y_{k} \geq 0, \quad \forall i, S, k
\end{gathered}
$$

where new variables $k \in K$ are introduced which correspond to a partition of items in to bundles. $K$ is the set of all partitions. $y_{k}=1$ if auctioneer chooses $k^{\text {th }}$ partition, $y_{k}=0$ otherwise. Bikchandani and Ostroy prove the optimal feasible solution to the above linear programme is integral and solve the Integer Programming formulation of combinatorial resource allocation problem.

\section{Dual}

$$
\min _{P_{i}, R_{i S}, \pi} \sum P_{i}+\pi
$$

sub. to

$$
\begin{gathered}
P_{i}+R_{i S} \geq M-V_{i S}, \quad \forall i, S \\
\pi-\sum_{[i, S] \in k} R_{i S} \geq 0, \quad \forall k
\end{gathered}
$$




$$
P_{i}, R_{i S}, \pi \geq 0
$$

where

$$
R_{i S}=M-P_{i S}
$$

Let $S_{i}$ denote the provisional allocation to bidder $i$, and $P_{i S}^{a s k}$ denote the price auctioneer wants to pay for bundle $S$ to agent $i$.

Feasible Primal: To construct a feasible primal solution assign $x_{i S_{i}}=1$ and $x_{i S^{\prime}}=0$ for all $S^{\prime} \neq S_{i}$. Partition $y\left(k^{*}\right)=1$ for $k^{*}=\left[S_{1}, \ldots, S_{|I|}\right]$, and $y_{k}=0$ otherwise.

Feasible Dual: To construct a feasible dual solution assign $P_{i S}=P_{i S}^{a s k}$.

The value $P_{i}$ can be interpreted as bidder $i$ 's max. utility at the prices and $\pi$ can be interpreted as min. amount, auctioneer has to pay for the set of items $G$. Of course the auctioneer can not solve explicitly the value $P_{i}$ since $V_{i S}$ are the private values of the bidders.

We can understand $P_{i s k}^{a s k}=P_{i S}$ means auctioneer giving discriminatory prices to agents for getting bundle $S$. If the prices are nondiscriminatory then

$P_{i S}=P_{j S} \forall j \in I$

we can formulate a new linear program, whose dual has non discriminatory prices. We call that as linear program-2 (LP-2)

\section{LP-2 Primal}

$$
\max _{i S}, y_{k} \sum_{S \subseteq G} \sum_{i \in I} x_{i S}\left(M-V_{i S}\right)
$$

sub. to

$$
\begin{gathered}
\sum_{S \subseteq G} x_{i S} \leq 1, \quad \forall i \\
\sum_{i \in I} x_{i S} \leq \sum_{i} k \in K, S \in k y_{k}, \quad \forall S \\
\sum_{k \in K} y_{k} \leq 1 \\
x_{i S}, y_{k} \geq 0, \quad \forall i, S, k
\end{gathered}
$$

LP-2 Dual

$$
\min _{P_{i}, R_{S}, \pi} \sum P_{i}+\pi
$$

sub. to

$$
P_{i}+R_{S} \geq M-V_{i S}, \quad \forall i, S
$$

$$
\pi-\sum_{[i, S] \in k} R_{S} \geq 0, \quad \forall k
$$

$$
P_{i}, R_{S}, \pi \geq 0
$$

where

$$
R_{S}=M-P_{S}
$$

$$
P_{i}+\left(M-P_{S}\right) \geq M-V_{i S}, \quad \forall i, S
$$

$$
\pi-\sum_{[i, S] \in k}\left(M-P_{S}\right) \geq 0, \quad \forall k
$$

$$
\pi-M^{1}+\sum_{[i, S] \in k}\left(P_{S}\right) \geq 0, \quad \forall k
$$

$$
\pi \geq M^{1}-\sum_{[i, S] \in k^{-}}\left(P_{S}\right)
$$

$$
\pi=\max \left[M^{1}-\sum_{[i, S] \in k}\left(P_{S}\right)\right]
$$

$$
\pi=\min \left[\sum_{[i, S] \in k}\left(P_{S}\right)\right]
$$

Claim by Bikchandani and Ostroy: If

(1) $\mathrm{V}\left(S_{1} \cup S_{2}\right) \geq \mathrm{V}\left(S_{1}\right)+\mathrm{V}\left(S_{2}\right) \forall$ non conflicting bundles i.e. $S_{1} \cap S_{2}=\phi$

(2) Agents demand bundles from the same partition $k$, then $V^{*}{ }_{L P-2}=\mathrm{V}^{*}{ }_{I P}$

$\left(\mathrm{P}_{i}, \pi\right)$ is the dual feasible solution.

\subsection{Iterative Auction Algorithm (iBun- dle)}

Auction starts by initiating $P_{S}^{a s k}=M$ for all bundles $S$ and at each round auctioneer decreases $\epsilon$ amount for all bundles which are over demanded and minimize $\pi$ (equation 27) by provisionally allocating bundles to the bidders (tentative winners) which is nothing but evaluating feasible primal solution. The iterative process progress until the feasible primal and dual solutions satisfy complementary-slackness conditions ( refer Parkes thesis [1] ). 

tions

$$
\begin{array}{lr}
\text { CS-1 } x_{i S}>0 \Rightarrow \mathrm{P}_{i}+P_{S}=V_{i S}, & \forall \mathrm{i}, \mathrm{S} \\
\text { CS-2 } y_{k}>0 \Rightarrow \pi-\sum_{S \in k} P_{S}=0, & \forall \mathrm{k} \\
\text { CS-3 } \mathrm{P}_{i}>0 \Rightarrow \sum_{S \subseteq G} x_{i S}=1, & \forall \mathrm{i} \\
\text { CS-4 P }>0 \Rightarrow \sum_{i \in I} x_{i S}=\sum_{k \in K, s \in k} y_{k}, & \forall \mathrm{S} \\
\text { CS-5 } \pi>0 \Rightarrow \sum_{k \in K} y_{k}=1 &
\end{array}
$$

Condi-
While auction is progressing on, algorithm strives to satisfy the above complementary-slackness conditions. Except CS-3, all other CS conditions will be satisfied in all rounds of the auction. CS-3 will be achieved at the termination of the auction.

\subsection{Winner Determination (WD) Prob- lem}

At each round auctioneer announces ask prices $\left(P_{S}^{a s k}\right)$ for all bundles $\mathrm{S}$. After knowing the ask prices the bidders send their bids. If the response from bidder $i$ is less than or equal to the ask price of the bundle we consider that bid as a best response bid $\left(P_{S_{i}}^{b e s t}\right)$ and $B_{i}$ is the set of all best response bids from the agent $i$. At each and every round auctioneer has to solve an allocation problem using best response bids for getting tentative winners.

$$
\min _{S_{1}, \ldots, S_{I}} \sum_{i \in I} P_{S_{i}}^{\text {best }}
$$

sub. to

$$
\begin{gathered}
S_{i} \cap S_{j}=\phi, \quad \forall i, j \\
P_{S_{i}}^{\text {best }} \in B_{i}
\end{gathered}
$$

Provisional allocation (for getting all items in set $G$ ) $S^{*}=\left(S_{1}^{*}, S_{2}^{*}, \ldots, S_{I}^{*}\right)$

At every round of the auction, the auctioneer has to solve the WD problem (finding tentative winners for minimizing $\pi$ ), which is an NP-hard problem. The size of the problem depends on the number of best response bids after decreasing price $\epsilon$ on the bundles. By increasing the $\epsilon$ value one can avoid more number of bids, but there is a loss in economy. The example given bellow discusses a simple reverse'auction for two items $A, B$ to be procured from three bidders $A_{1}^{b}, A_{2}^{b}, A_{3}^{b}$. The ask prices on possible bundles are $P_{A}^{a s k}, P_{B}^{a s k}, P_{A B}^{a s k}$. R, TA, C, $U^{b}$ are auction round, tentative allocation, Cost to be paid for the tentative allocation and number of unsuccessful bids respectively. In the example we choose $\epsilon=5$ for all rounds of the auction. Instead of a fixed $\epsilon$, if we can reach the end of the auction in less number of rounds by choosing a variable $\epsilon$, which takes different values at different rounds with out loss of economy, then we can reduce the computational cost for the procurement process. One can reduce the computational cost by taking large values for $\epsilon$ 's but may not get best response bids so auctioneer has to pay high price (economy loss ). Bidder $i$ 's private valuation for bundle $S\left(V_{i}\right)$ vary from auction to auction (nondeterministic value). In the next section we formulate a stochastic dynamic programming problem, which has reward function in terms of cost reduction from one round to the next round by choosing an $\epsilon$ at a round of auction and computational cost in the next round by choosing the $\epsilon$. For minimizing the reward function we have to find optimal policy (best sequence of $\epsilon^{\prime}$ s ).

We would like to answer the above problem by using Reinforced-Learning for choosing $\epsilon$.

\section{Reinforced-Learning for Choosing $\epsilon$}

Reinforcement Learning (RL) procedures are practical methods for solving Markov Decision Problems. Actor-critic algorithm is an algorithm for learning to estimate the long-term expected reward for a given state. It does not need the model of the environment (in our problem environment is the non deterministic private values $V_{i S}$ of bidders ), and it can be used for online learning. If the state space is large where look up tables are infeasible, RL methods can be combined with function approximators to set good practical results as in the Neuro Dynamic Programming (NDP) [4]

State space $=$ number of best response bids $(B)$, cost to be paid for set of items $G$ at that moment $(C)$

$\operatorname{Reward}(r)=\left(C_{t}-C_{t+1}\right)-\mathrm{f}\left(B_{t+1}\right)$

where $B_{i}$ is the number of best response bids at state $i$.

Let $\epsilon$ be the decrement, the auctioneer will choose on all over demanded bundles for the next round bids. That means the auctioneer chooses one of the possible controls from the set

$E=\left\{\epsilon_{1}, \epsilon_{2}, \ldots\right\}$

If the probability structure is known, then we can solve the above stochastic dynamic programming by backward induction. When the probability structure is not known a priori, Reinforcement Learning in MDPs can be used to learn an optimal Markovian determine

$j$ and $g(i, u, j)$ are generated from the pair $(i, u)$ by simulation.

\section{Conclusions}

In this paper, we have proposed an efficient auction mechanism for e-procurement, which we call IBPA (Iterative Bundle Procurement Auction). IBPA is based 
Table 1: an example for e-Procurement auction

\begin{tabular}{l|l|l|l|l|l|l|l|l|l}
\hline \hline $\mathrm{R}$ & $\mathrm{P}_{A}^{a s k}$ & $\mathrm{P}_{B}^{a s k}$ & $\mathrm{P}_{A B}^{a s k}$ & $\mathrm{~A}_{1}^{b}$ & $\mathrm{~A}_{2}^{b}$ & $\mathrm{~A}_{3}^{b}$ & $\mathrm{TA}$ & $\mathrm{C}$ & $\mathrm{U}^{b}$ \\
\hline 1 & 100 & 100 & 200 & $(\mathrm{AB}, 200)$ & $(\mathrm{A}, 100),(\mathrm{AB}, 200)$ & $(\mathrm{B}, 100),(\mathrm{AB}, 200)$ & {$[2, \mathrm{AB}]$} & 200 & 2 \\
2 & 100 & 95 & 195 & $(\mathrm{AB}, 195)$ & $(\mathrm{A}, 100),(\mathrm{AB}, 195)$ & $(\mathrm{B}, 95),(\mathrm{AB}, 195)$ & {$[1, \mathrm{AB}]$} & 195 & 2 \\
3 & 95 & 90 & 190 & $(\mathrm{AB}, 195)$ & $(\mathrm{A}, 95),(\mathrm{AB}, 190)$ & $(\mathrm{B}, 90),(\mathrm{AB}, 190)$ & {$[2, \mathrm{~A}],[3, \mathrm{~B}]$} & 185 & 1 \\
4 & 95 & 90 & 185 & $(\mathrm{AB}, 185)$ & $(\mathrm{A}, 95),(\mathrm{AB}, 185)$ & $(\mathrm{B}, 90),(\mathrm{AB}, 190)$ & {$[2, \mathrm{AB}]$} & 185 & 2 \\
$\vdots$ & $\vdots$ & $\vdots$ & $\vdots$ & $\vdots$ & $\vdots$ & & & & \\
& & & & & & & $\vdots$ \\
last but one & 45 & 40 & 80 & $(\mathrm{AB}, 80)$ & $(\mathrm{A}, 50),(\mathrm{AB}, 90)$ & $(\mathrm{B}, 40),(\mathrm{AB}, 90)$ & {$[1, \mathrm{AB}]$} & 80 & 1 \\
last round & 45 & 35 & 80 & $(\mathrm{AB}, 80)$ & $(\mathrm{A}, 50),(\mathrm{AB}, 90)$ & $(\mathrm{B}, 40),(\mathrm{AB}, 90)$ & {$[1, \mathrm{AB}]$} & 80 & 0 \\
\hline
\end{tabular}

on the iBundle algorithm [1]. We have developed a reinforcement learning approach to orchestrate the iterations of IBPA in a way to improve the computational efficiency of the iterative algorithm.

We believe IBPA will lead to achieving efficiencies in e-procurement. We need to look into many issues before applying IBPA in real-world settings. These issues include: design of bidding logic for buyer and the sellers; quantifying the algorithmic efficiency of IBPA; and application to the case where multiple units of multiple items are to be procured.

\section{References}

[1] Parkes, D. C, Iterative Combinatorial Auctions, Doctoral Dissertation, Computer and Information Science, University of Pennsylvania, 2001

[2] Bickchandani, S and Ostroy, J. M, The Package assignment model, Technical report, UCLA

[3] Bertsekas, D, Dynamic Programming and Optimal Control, Vol 2, Athena Scientific, Massachusetts, 1995

[4] Bertsekas, D, and Tsitsiklis; J. N. Neuro-Dynamic Programming, Athena Scientific, Massachusetts, 1996

[5] Greenwald, A. R and Kephart, J. O. Shopbots and Pricebots, Proceedings of the International Joint Conference on Artificial Intelligence, IJCAI99, pp:506-511, 1999

[6] Ravikumar, K and Manish Gupta, Reinforcement Learning Algorithms for Price Control in a Dutch Auction, Technical Report, IBM India Research Laboratory, New Delhi, India, 2001

[7] Tesauro, G and Kephart, J. O. Pricing in Agent Economies using Multi-agent Q-learning, Proceedings of the Workshop on Game Theoretic and Decision Theoretic Agents, 1999
[8] Watkins, C. J. C. H and Dayan, P. Q-learning, Machine Learning, 8, pp 279-292, 1992

[9] Nils, J. N, Introduction to Machine Learning, 1996

[10] Roy, B. V, Bertsekas, D. P, Lee, Y and Tsitsiklis, J. N. A Neuro-Dynamic Programming Approach to Retailer Inventory Management, LIDS, MIT, 1999

[11] Junling, H, Daniel, R and Wong, H. Agents Participating in Internet Auctions, AIL, University of Michigan, 1999

[12] McAfee, R. P, and McMillan. Auctions and Bidding, Journal of Economic Literature, vol. 25, pp. 699-738, june 1987

[13] Ron, S and Chad, S. Reinforcement Learning with Bidding for Automatic Segmentation

[14] Nison, N. Bidding and Allocation in Combinatorial Auctions, ICS, HU, Jarusalem, 2000

[15] Varies, S. D and Vohra, R. Combinatorial Auctions: A Survey, MEDS, Kellogg School of Management, Northwestern University, 2000 